\title{
Clinical Trial Recruitment Plan
}

National Cancer Institute

\section{Source}

National Cancer Institute. Clinical Trial Recruitment Plan. NCI Thesaurus. Code C115769.

A proposed method to describe subject enrollment and recruitment goals during a clinical trial. 